\title{
Novelty Detection with One-Class Support Vector Machines
}

\author{
John Shawe-Taylor and Blaž Žličar
}

\begin{abstract}
In this paper we apply one-class support vector machine (OC-SVM) to identify potential anomalies in financial time series. We view anomalies as deviations from a prevalent distribution which is the main source behind the original signal. We are interested in detecting changes in the distribution and the timing of the occurrence of the anomalous behaviour in financial time series. The algorithm is applied to synthetic and empirical data. We find that our approach detects changes in anomalous behaviour in synthetic data sets and in several empirical data sets. However, it requires further work to ensure a satisfactory level of consistency and theoretical rigour.
\end{abstract}

Keywords Financial time series • Novelty detection • One-class SVM

\section{Introduction}

We apply one-class support vector machine (OC-SVM) to synthetic and empirical data and test its ability to detect anomalous behaviour in a time series. Anomalous behaviour in this case is a combination of consecutive data points in a time series that do not belong to a distribution identified by the algorithm. We first briefly introduce the theory behind the OC-SVM. Then we present its application to novelty detection in a time series by using lagged returns as inputs. Results, main conclusions and recommendations for further research are outlined at the end.

\footnotetext{
J. Shawe-Taylor • B. Žličar $(\bowtie)$

Department of CS, University College London, London, UK

e-mail: j.shawe-taylor@cs.ucl.ac.uk; b.zlicar@cs.ucl.ac.uk
} 


\section{Background: Novelty Detection and One-Class SVM}

We begin by quoting a result from [1] that bounds the likelihood that data generated according to the same distribution used to train OC-SVM will generate a false alarm.

Theorem 1 Fix $\gamma>0$ and $\delta \in(0,1)$. Let $(\mathbf{c}, r)$ be the centre and radius of $a$ hypersphere in a feature space determined by a kernel $\kappa\left(\mathbf{x}, \mathbf{x}^{\prime}\right)=\left\langle\phi(\mathbf{x}), \phi\left(\mathbf{x}^{\prime}\right)\right\rangle$ from a training sample $S=\left\{\mathbf{x}_{1}, \ldots, \mathbf{x}_{\ell}\right\}$ drawn randomly according to a probability distribution $\mathscr{D}$. Let $g(\mathbf{x})$ be the function defined by

$$
g(\mathbf{x})= \begin{cases}0, & \text { if }\|\mathbf{c}-\boldsymbol{\phi}(\mathbf{x})\| \leq r ; \\ \left(\|\mathbf{c}-\boldsymbol{\phi}(\mathbf{x})\|^{2}-r^{2}\right) / \gamma, & \text { if } r^{2} \leq\|\mathbf{c}-\boldsymbol{\phi}(\mathbf{x})\|^{2} \leq r^{2}+\gamma \\ 1, & \text { otherwise. }\end{cases}
$$

Then with probability at least $1-\delta$ over samples of size $\ell$ we have

$$
\mathbb{E}_{\mathscr{D}}[g(\mathbf{x})] \leq \frac{1}{\ell} \sum_{i=1}^{\ell} g\left(\mathbf{x}_{i}\right)+\frac{6 R^{2}}{\gamma \sqrt{\ell}}+3 \sqrt{\frac{\ln (2 / \delta)}{2 \ell}},
$$

where $R$ is the radius of a ball in feature space centred at the origin containing the support of the distribution.

Hence, the support of the distribution outside the sphere of radius $r^{2}+\gamma$ centred at $\mathbf{c}$ is bounded by the same quantity, since $g(\mathbf{x})=1$ for such inputs and is less than 1 elsewhere. Note moreover that the function $g(\mathbf{x})$ can be evaluated in kernel form if the optimisation is solved using its dual.

The theorem provides the theoretical basis for the application of the OC-SVM and it is perhaps worth dwelling for a moment on some implications for time-series analysis.

- Firstly, the assumption made by the theorem about the distribution $\mathscr{D}$ generating the training and test data has strong and weak elements:

- It is strong in the sense that there are no assumptions made about the form of the input distribution $\mathscr{D}$. It therefore applies equally to long-tailed distributions as it does to multivariate Gaussians. We will perform some experiments with real-world data in which any assumptions about the form of the generating distribution would be difficult to justify.

- It is weak in the sense that it assumes the training data are generated independently and identically (i.i.d.), something that will not to be strictly true for time series. This assumption becomes more reasonable when the training data are drawn from separate parts of the time series. 
- The theorem is one sided in that it bounds the probability that data that arose from the training distribution are mistaken for novel outliers. It does not, however, say anything about the likelihood that novel data are not detected.

In connection with the final point, Vert and Vert [3] provide an interesting analysis showing that if we generate negative data from an artificial background measure $\mu$ and train as a 2-class SVM, in the limit of large data the SVM will identify the level sets of the pdf of the training distribution relative to $\mu$. This suggests that it finds the minimal density with respect to $\mu$ consistent with capturing a given fraction of the input distribution. Hence, in this case, we can make assertions about the efficiency with which outliers are detected.

\section{Problem: Novelty Detection in Financial Time Series}

In order to apply OC-SVM to a single time series we follow the approach proposed by Ma and Perkins [2]. We extend this approach by adding an exponential decay parameter so that the more recent lags carry more weight than the older lags, since we are interested in detecting anomalies in the very short window before the occurrence of the extreme volatility, the underlying hypothesis being that the behaviour of the market changes before the occurrence of the spike in the time series.

\subsection{Data Preprocessing}

A data matrix is constructed in such a way so that the first column represents the original time series and every next column is a lag of the previous column. More specifically, for a time series variable $x$ composed of observations $x(t)$ where $t=$ $1, \ldots, T$ ( $T$ being the number of time points, observations) we perform a vectorto-matrix transformation so that the dimensionality of the original column vector $\mathbf{x}$ changes from $T x l$ to $(T-d-1)$ x $d$ forming a data matrix $X$. Here $d$ represents our choice of the dimensionality expansion, i.e. the number of all columns in the newly formed matrix $X$ in effect reflecting the number of lags we chose to include in the analysis. Alternatively, we can think of this in terms of extending the dimensionality of a data point $x(t)$ to a row vector $\mathbf{x}(\mathrm{t})$ so that

$$
\mathbf{x}(t)=[x(t) \ldots x(t-d+2) x(t-d+1)]
$$

Then the newly formed data matrix in terms of row vectors becomes

$$
X=[\mathbf{x}(d), \ldots, \mathbf{x}(T)]^{\top}
$$


with dimensions (T- $d-1)$ x $d$ (as suggested by Ma and Perkins [2]). We then take a step further and add a decay parameter $c$ so that the weight of each next column falls exponentially with each lag. If we denote a row vector $\mathbf{d}=[1, \ldots, d]$ then we define $c^{\mathbf{d}}$ to be the row vector

$$
\mathbf{c}=c^{\mathbf{d}}=\left[c^{1}, \ldots, c^{d}\right]
$$

where $c$ is an arbitrarily chosen decay parameter $0<c<1$. The new data matrix taking into account the exponential time decay is then

$$
X_{c}=X \odot D
$$

where $D$ is a matrix of decay factors $D=\mathbf{1}^{\top} * \mathbf{c}$ and multiplication between $X$ and $D$ is element by element multiplication. Alternatively, if we denote the number of columns in $X$ as $j=1, \ldots, d$, then we can simply define the matrix $D$ as having entries $D_{i j}=c^{j} . X_{c}$ is then centred row-wise in a standard manner using a centering matrix $C$

$$
C=I_{d}-\frac{1}{d} O_{d}
$$

so that the final centred data matrix with a time decay is:

$$
X_{c}^{c}=X_{c} C
$$

\subsection{Novelty Detection Algorithm}

In this section we present a step-by-step pseudo-algorithm of OC-SVM based novelty detection in time-series analysis.

Input: a time series $x(t)$ of length $T$. Output: points in time identified as novelties.

(1) Vector-matrix transformation: Calculate $X_{c}^{c}$ using a range of different lags $d=[2: 20]$ to obtain 19 matrices of different dimensions $X E=\left[X_{2}^{c} \ldots X_{20}^{c}\right]$. The value of the decay parameter is set arbitrarily at $c=0.97$.

(2) Data sets: Each $X_{E}^{c}$ is split in a train set (X-train) of length $\frac{2}{3} T$ and the remaining third of observations comprise a test set (X-test). Further split $X$-train in half, that is into a $s u b$ - $X$-train and a val-X-train set.

(3) Train OC-SVM: Apply OC-SVM to sub-X-train so as to obtain $\alpha_{i}$ for each $X_{c}^{c}$ in the array of matrices $X E=\left[X_{2}^{c} \ldots X_{20}^{c}\right]$ individually and for all values of 
$\gamma=2^{i}$ (where $\left.i=[-10: 10]\right)^{1}$ and $v=2^{j}$ where $(j=[-15:-1])$. Then find pseudo-optimal $d_{o}, \gamma_{o}$ and sensitivity parameter $v_{o}$ by locating the OC-SVM that achieved the highest accuracy on val-X-train. ${ }^{2}$

(4) Test optimal OC-SVM $\left(d_{o}, \gamma_{o}, v_{o}\right)$ : Use pseudo-optimal values determined in (3) to train OC-SVM $\left(d_{o}, \gamma_{o}, v_{o}\right)$ on $X$-train. Test the model on $X$-test and obtain the novelty signal for the test set.

\section{Experiments}

Firstly, we describe the construction of synthetic time series and present the empirical data sets (three stock market indices). Next, we comment on the results and outline the challenges.

\subsection{Data}

Both synthetic and empirical data sets are of about the same length $(T \approx$ 5800). Synthetic time series are comprised of an original signal in the training set while in the test sets we add anomalies (i.e. time intervals where the original time series is corrupted by an anomalous signal) on the intervals $T=$ [5000:5050, 5300:5350, 5600:5650]. We train the OC-SVM algorithm on a data set comprised solely of original (non-anomalous) time series and then test the optimal specification of the model on a test set that includes pre-defined intervals with anomalies. Synthetic time series are constructed as follows:

$$
x(t)=\left\{\begin{array}{l}
x_{a}(t) \text { for } t \in[5000,5050] \wedge[5300,5350] \wedge[5600,5650] \\
x_{o}(t) \text { for } t \notin[5000,5050] \wedge[5300,5350] \wedge[5600,5650]
\end{array}\right.
$$

Here $x_{o}(t)$ denotes the original time series and $x_{a}(t)$ denotes the anomalous time series. Synthetic data sets are then the following three time series types:

1. Synthetic 1 time series is a sinusoid with a small standardised random noise in the training set, but with increased standard deviation of the error process on

\footnotetext{
${ }^{1}$ We use radial basis kernel (RBF) so that $k(\mathbf{x}, \mathbf{y})=\exp \left(-\gamma\|\mathbf{x}-\mathbf{y}\|^{2}\right)$.

${ }^{2}$ We use a term pseudo-optimal since we simply choose a specification that is able to contain all training data and consequently label sub-X-train data sample as novelty-free. Clearly, this is not necessarily the optimal solution nor is it the only solution and presents one of the main challenges related to novelty detection with OC-SVM.
} 
specific intervals within the test set. The synthetic 1 signal follows

$$
x_{1}(t)=\sin \left(\frac{40 \pi}{N}\right)+\epsilon(t)
$$

where

$$
\epsilon(t)=\sigma z(t)
$$

and $z(t) \sim N(0,1)$ with sigma term in the error process equal to $\sigma_{o}=0.1$ for the original signal $x_{o}$ and a slightly higher sigma term $\sigma_{a}=0.2$ for the anomalous signal $x_{a}$.

2. Synthetic 2 time series is constructed as a random process taking into account the empirical (sample) mean and standard deviation of an empirical time series (in our case the S\&P500 stock market index). The original time series is constructed by adding an error term sampled from a standardised normal distribution and the anomalous signal is obtained by adding an error noise sampled from a student- $t$ distribution. We write the original time series as

$$
x_{2}(t)=\mu+\epsilon(t)
$$

where

$$
\epsilon_{o}(t)=\sigma_{S P 500} z(t)
$$

and $z(t) \sim N(0,1)$.

The anomalous signal follows the same process only with its noise term sampled from student-t distribution with six degrees of freedom

$$
\epsilon_{a}(t)=\sigma_{S P 500} z(t)
$$

and $z(t) \sim t_{6}$.

3. Synthetic 3 time series is obtained by subtracting the mean from the synthetic 2 signal and then taking the absolute value of the obtained time series. Such absolute returns are often used as a proxy for a volatility process in financial research. In other words, the synthetic 3 signal is equal to absolute error term in Eq. (10).

$$
x_{3}(t)=|\epsilon(t)|=\left|\sigma_{S P 500} z(t)\right|
$$

with $z(t) \sim N(0,1)$ for the original time series and $z(t) \sim t_{6}$ for the anomalous time series.

Empirical data sets are time series of three stock market indices: S\&P500, DAX30 and NIKKEI225. We work with adjusted daily closing prices obtained 
from Yahoo.Finance for a period of approximately 13 years, where we perform the following two transformations of the original series:

1. Log returns are calculated by obtaining the difference between natural logarithms of a price at time $t$ and price at time $t-1$ :

$$
r(t)=\ln \left(\frac{p_{t}}{p_{t-1}}\right)
$$

Then we subtract the mean of the return time series:

$$
r_{\mathrm{dm}}(t)=r(t)-\mu
$$

2. Absolute returns are obtained by simply taking the absolute value of the log returns. Absolute returns are a frequently used proxy for a volatility measure in financial research

$$
r_{\mathrm{abs}}(t)=|r(t)|
$$

\subsection{Results}

In this section we describe the results for OC-SVM algorithm applied to synthetic and empirical data sets without and with the exponential decay parameter in the preprocessing phase. The two algorithms are denoted with OC-SVM-ND (no decay) and OC-SVM-ED (exponential decay), respectively. Optimal model specification is indicated by adding optimal parameter values in brackets so that $\mathrm{OC}-\operatorname{SVM}\left(d_{o}, \gamma_{o}, v_{o}\right)$ denotes the specification of OC-SVM with optimal index values for the dimension (indicating number of lags), $\gamma$ in RBF kernel and $v$ parameter in OC-SVM. ${ }^{3}$ Please note that this is a naive pseudo-optimisation simply assuming that the best OCSVM is the one that is able to put a bound around the data in a training set. We use LIBSVM support vector machine toolbox. Finally, figures are moved to Appendix to prevent cluttering.

\footnotetext{
${ }^{3}$ Where the numbers refer to the index not the value itself. For example, OC-SVM-ND $(1,2,3)$ would denote the optimal specification of OC-SVM without the decay parameter, where the optimal lag dimension is the first dimension in the dimension array $d=[2: 20]$, i.e. $d_{o}=2$, the optimal $v$ refers to the second position in the $j$ array $j=[-15:-1]$, i.e. $v_{o}=2^{-14}$, and the optimal $\gamma$ refers to the third position in the $i$ array $i=[-10: 10]$, i.e. $\gamma_{o}=2^{-8}$.
} 


\subsubsection{Synthetic Data}

Synthetic time series are constructed so that we can investigate the performance and the ability of OC-SVM to detect novelties that were artificially inserted in the testing part of the various types of data sets. Ideally, no novelties would be detected in the valuation part of the training set. In the test set the best performance is the one detecting novelties in the previously determined anomalous intervals.

Figure 1 displays the results for valuation part of the training set on the left side (with synthetic 1 at the top and OC-SVM-ND novelty signal at the bottom) and the test set on the right side. It shows that the model has the ability to learn the original signal in the training set since it correctly detects no novelties (novelty signal is equal to 1 at all times). When the same model is applied to the test set we see that it correctly identifies areas where anomalous data have been added to the original signal (grey areas). However, it also falsely returns novelty signal where no novelties have been added to the original synthetic signal, indicating that this particular OCSVM specification is perhaps still too sensitive to outliers. Figure 2 shows results of the OC-SVM-ED (with exponential decay) applied to the same synthetic 1 time series. This model is also successful in identifying the anomalous areas in the test set with slightly lower number of false positives. The results for other two synthetic signals are displayed in Figs. 3, 4, 5, and 6. Both algorithms, without and with decay parameter, are able to detect the anomalous areas with a small number of false positives. Only Fig. 3 stands out as it displays a poor performance of OC-SVM-ND in the test set of a synthetic 2 time series.

\subsubsection{Empirical Data}

Our empirical experiments focus on whether or not the algorithm detects anomalies slightly before volatility spikes. Figures 7 and 8 display results of OC-SVM applied to absolute returns of the empirical time series without and with decay, respectively. Figure 9 shows the valuation part of a training sample on the left and test sample on the right side for the S\&P500 stock market index. Top row displays the time series of S\&P500, middle row the return time series and bottom row the novelty signal for OC-SVM-ND. In this case our algorithm detects two intervals as anomalous (around time points 600 and 1300). Figure 9 displays identical figure twice with the only difference being that the two charts in fifth and sixth row on the right side are magnified around the novelty point so as to show the case of early novelty detection (around point 600). However, the volatility spike around time point 1300 is not detected in advance. The same results in both of these volatility cases are obtained when OC-SVM is applied using the exponential decay parameter (Fig. 10). Also, the results are very similar when both types of algorithms (with and without the decay parameter) are applied to S\&P500 absolute returns time series. In case of the DAX30 index none of the two algorithms detect sudden increase in volatility in advance when applied to time series of returns (Figs. 11 and 12). When applied to absolute returns (Figs. 13 and 14) both algorithms (OC-SVM-ND and OC-SVM-ED) detect 
the second volatility spike in advance (around the time point 550) but their first detections (around the time point 350) temporally coincide with the volatility spike. In case of the NIKKEI225 index both OC-SVM-ND and OC-SVM-ED detect the biggest volatility increase in advance (around the time point 600) when applied to returns and absolute returns. However, both specifications also fail to detect in advance the second spike around the time point 1200, again when applied to either returns or absolute returns (Figs. 15, 16, 17 and 18).

\section{Conclusions and Further Research}

In this paper we investigate the application of the OC-SVM to novelty detection in financial time series. We add an exponential decay parameter when preprocessing the data to account for the reduced dependency related to older data points. We test the OC-SVM on synthetic data sets and find that our algorithm manages to consistently identify anomalous areas inserted artificially in our test sets. Building on these results we then apply the algorithm to empirical data, namely financial time series of three stock market indices: S\&P500, DAX30 and NIKKEI225. The idea is that the projection of the market data into the feature space effectively allows for an inspection of market patterns we would normally not detect in the input space. This means that in cases when anomalous behaviour in the markets (reflected in the change of the distribution of the time series) has preceded the spike, our algorithm might be able to detect these anomalies. However, when the spike in volatility is the result of an unexpected exogenous event, OC-SVM will not be able to alert the user in advance since the time series is not reflecting the impending risk. ${ }^{4}$ Our experiments to some extent support this reasoning as the results show instances where OC-SVM, with and without a decay parameter, detects novelties occurring before volatility spikes, but such results are by no means conclusive. The unsupervised nature of OC-SVM allows for the detection of previously unseen observation, however it simultaneously prevents us from targeting a type of process (event). This makes it useful for novelty detection in synthetic data sets (where novelty points are known in advance) while making it problematic for the application to empirical data sets. ${ }^{5}$ Optimisation of the hyperparameter $v$ in OCSVM is a challenge in itself and when applied to financial time-series analysis this problem becomes even more difficult. Future research could perhaps investigate a possible connection between $v$ and the level of randomness of the underlying time series. Also, it would be interesting to investigate the usefulness of one-class SVM for novelty detection in multivariate data.

\footnotetext{
${ }^{4}$ Note that for the synthetic data this does not arise since the volatility takes immediate effect.

${ }^{5}$ Especially when applied to extremely noisy data such as financial time series.
} 


\section{Appendix}
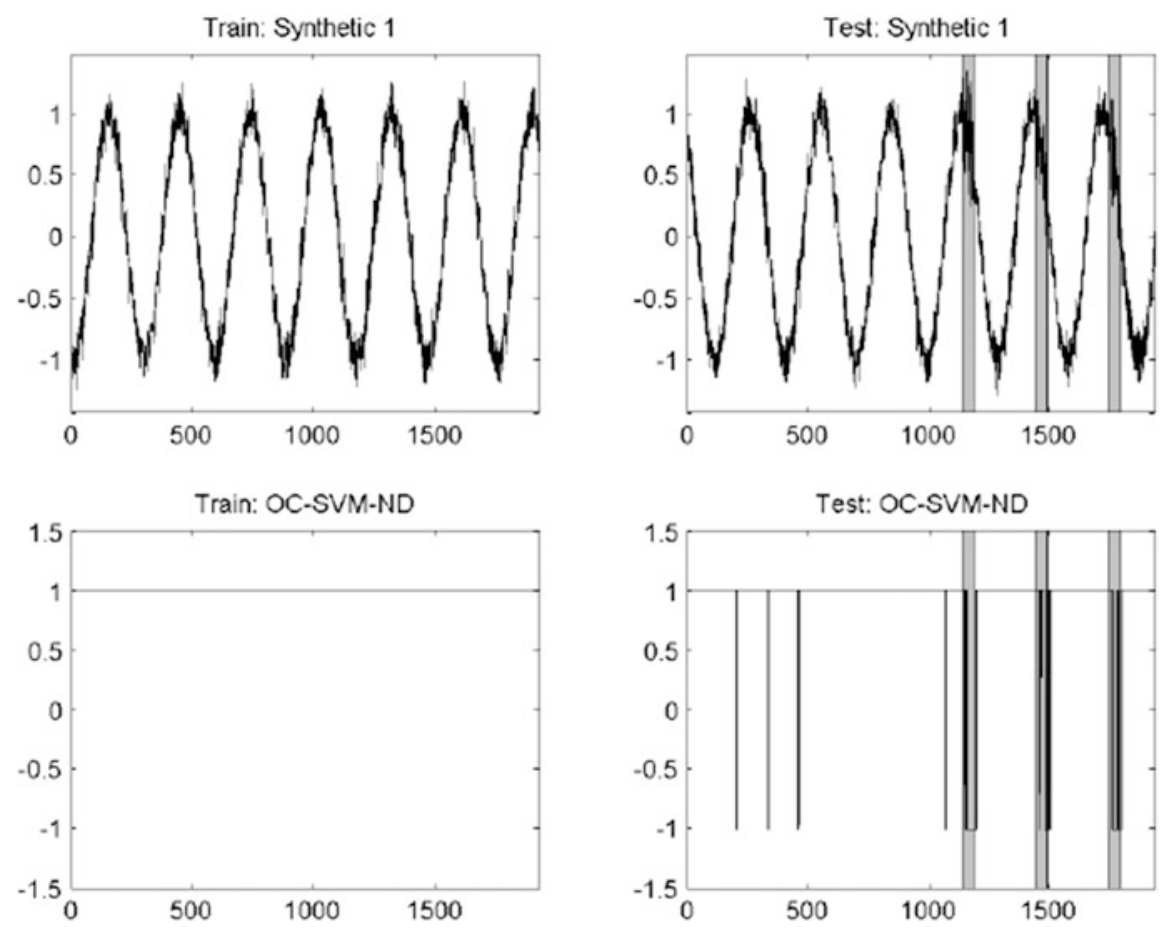

Fig. 1 Synthetic 1: OC-SVM-ND $(19,7,1)$ 
Train: Synthetic 1

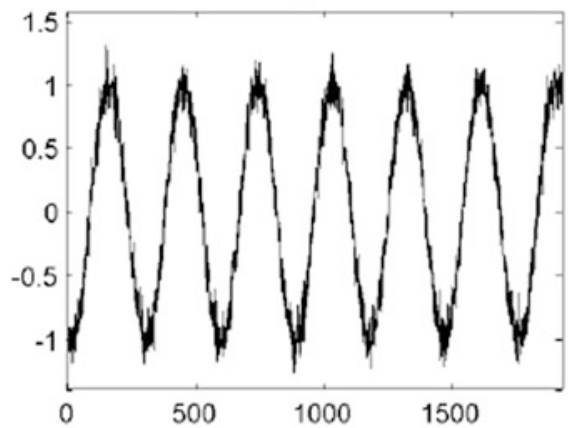

Train: OC-SVM-ED

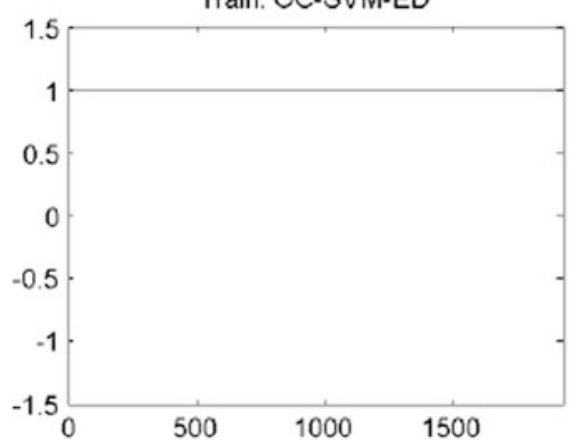

Fig. 2 Synthetic 1: OC-SVM-ED $(12,7,1)$
Test: Synthetic 1
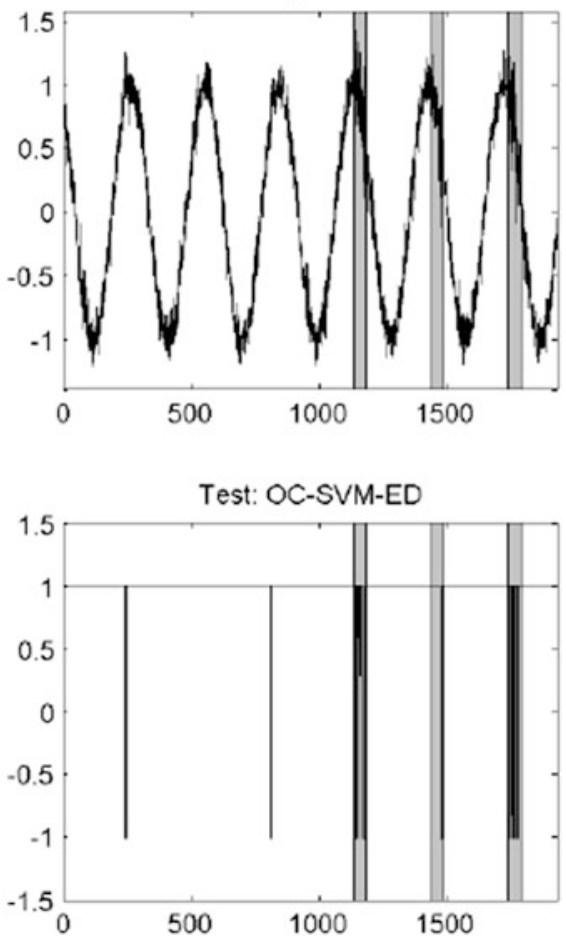

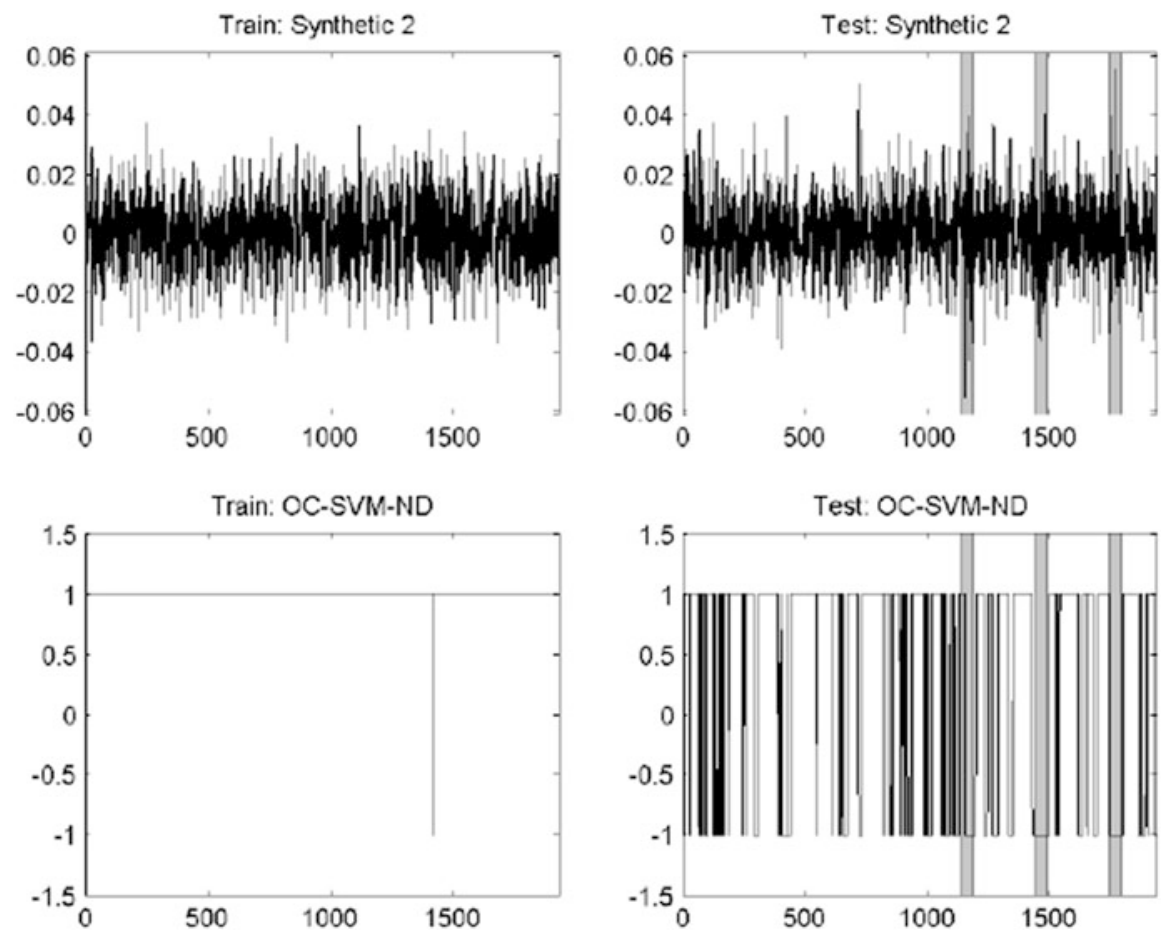

Fig. 3 Synthetic 2: OC-SVM-ND $(15,9,5)$ 

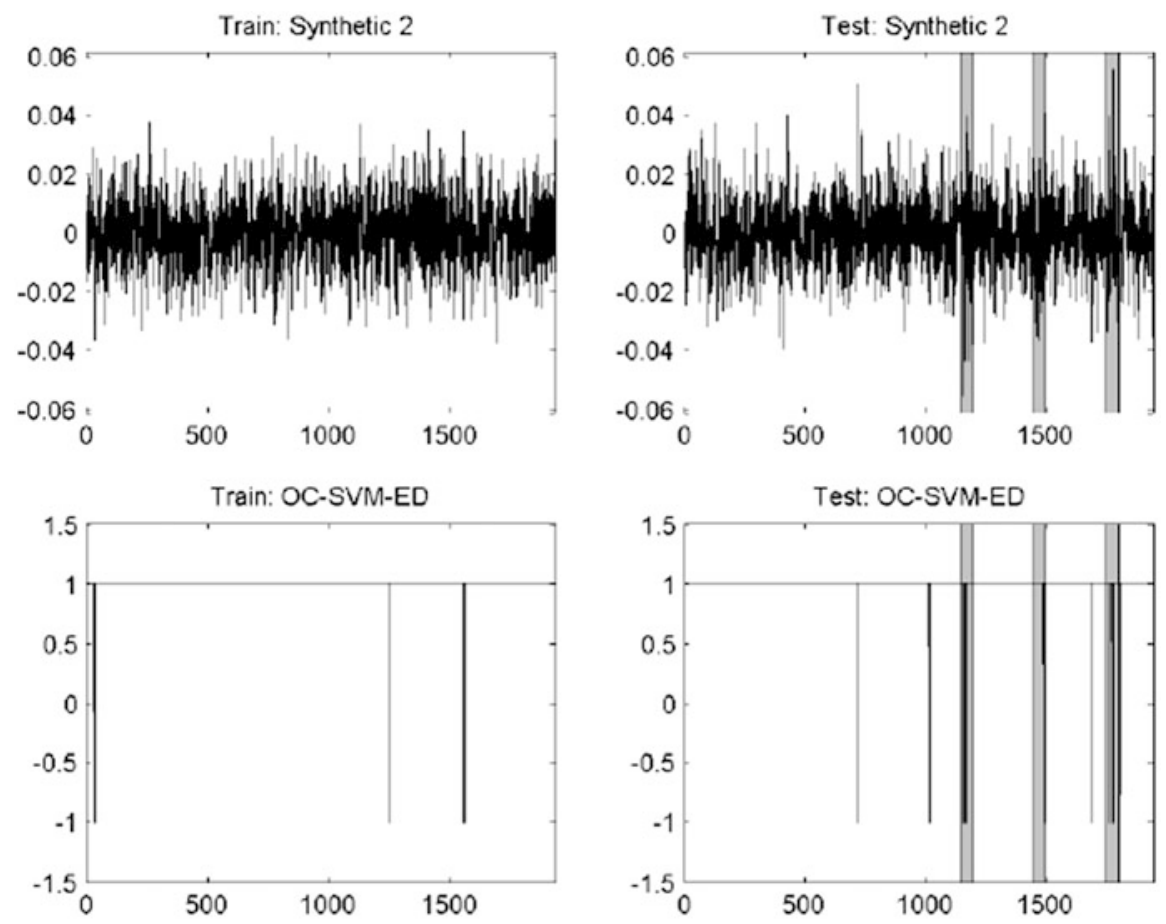

Fig. 4 Synthetic 2: OC-SVM-ED $(1,7,9)$ 
Train: Synthetic 3

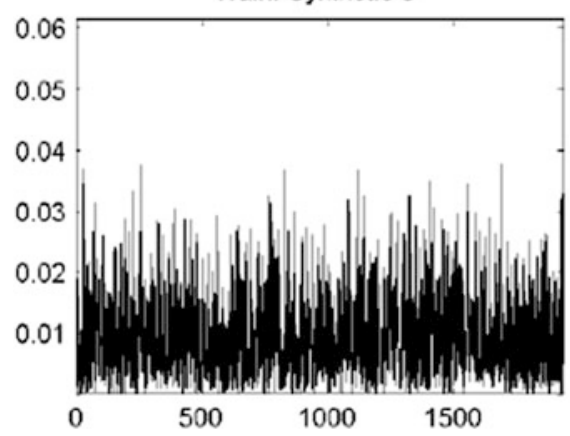

Train: OC-SVM-ND

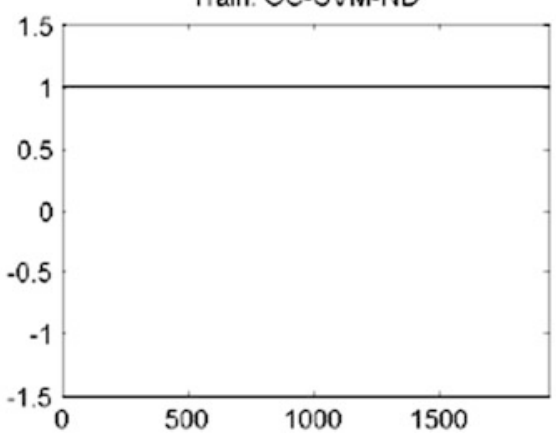

Test: Synthetic 3

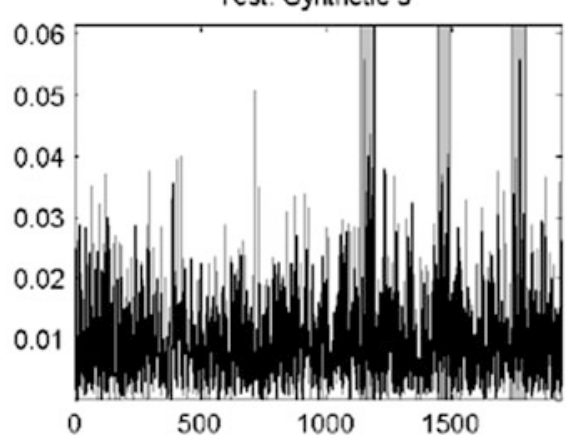

Test: OC-SVM-ND

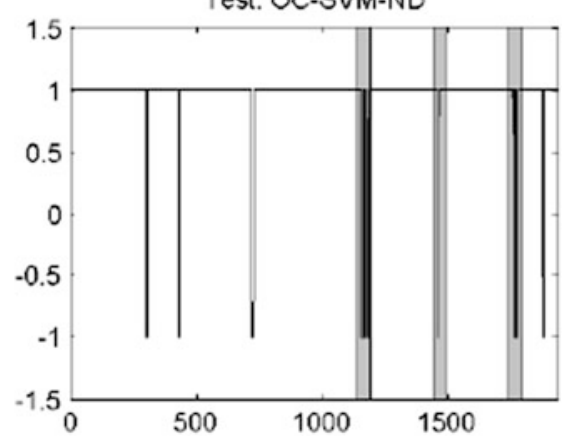

Fig. 5 Synthetic 3: OC-SVM-ND $(8,8,8)$ 

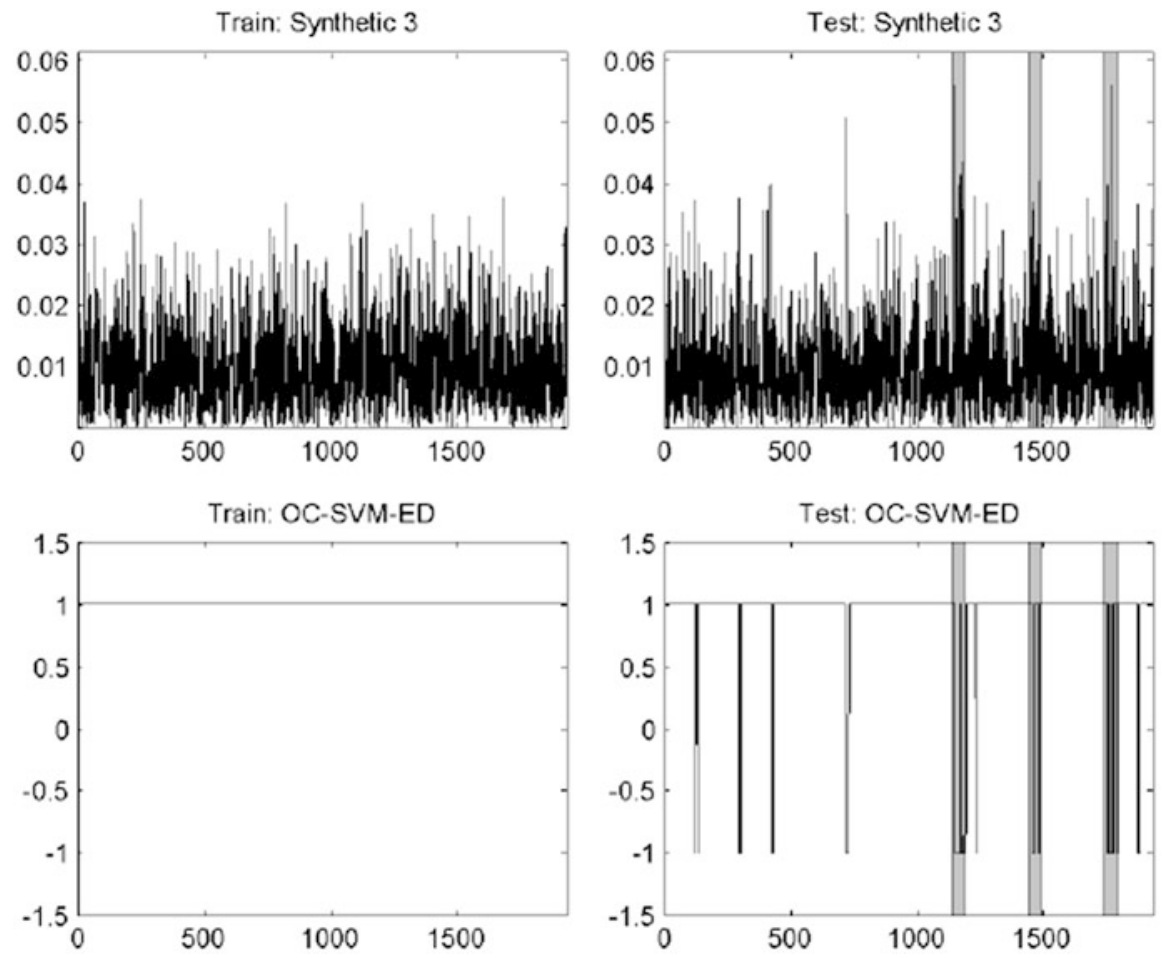

Fig. 6 Synthetic 3: OC-SVM-ED $(13,9,7)$ 

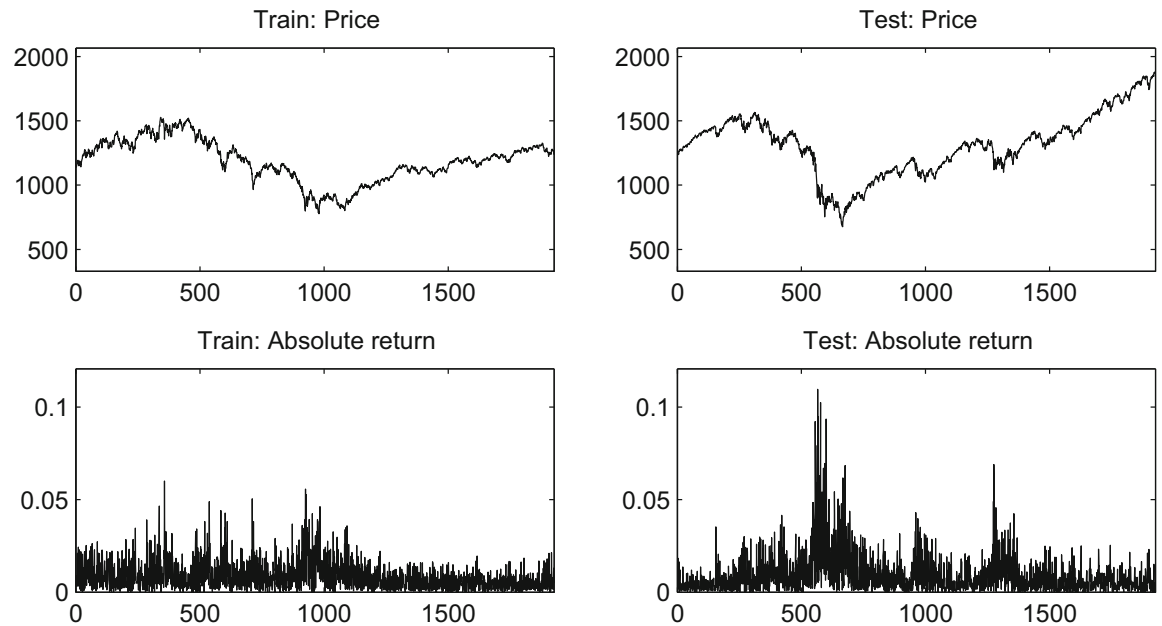

Train: OC-SVM-ND
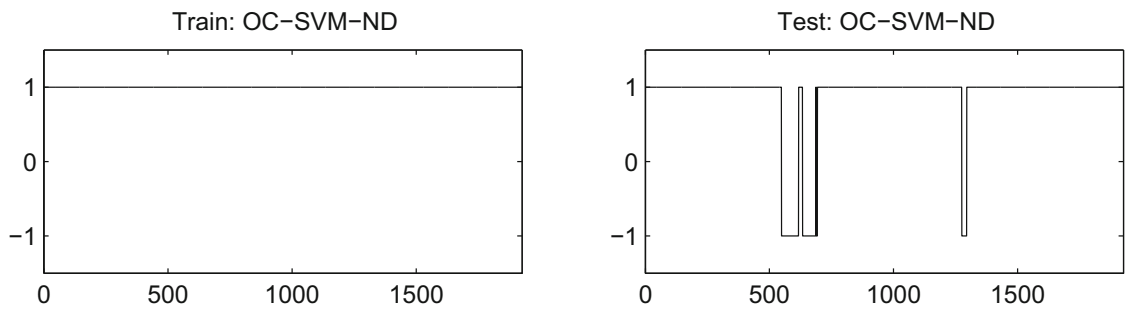

Fig. 7 S\&P500 absolute returns: OC-SVM-ND $(17,10,4)$ 

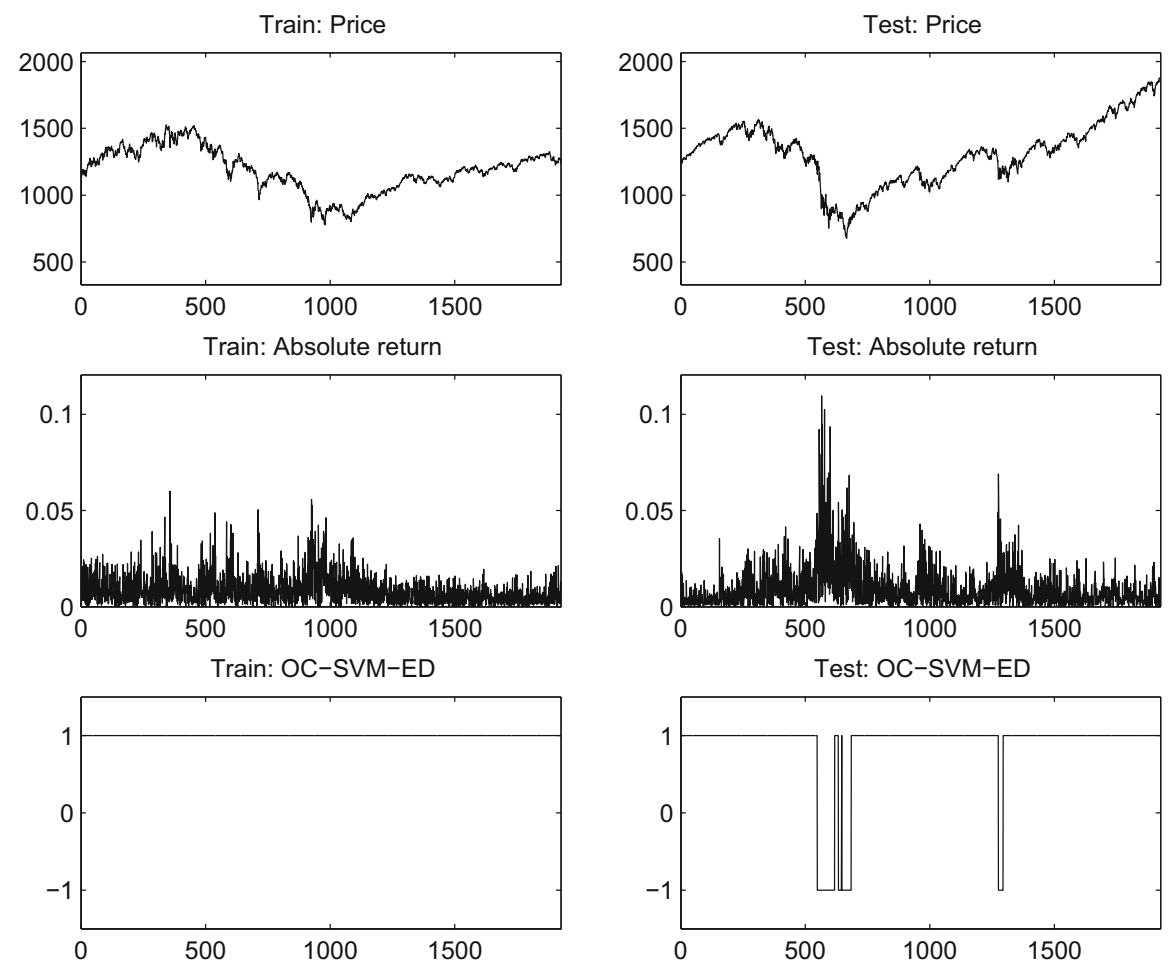

Fig. 8 S\&P500 absolute returns: OC-SVM-ED $(17,10,5)$ 

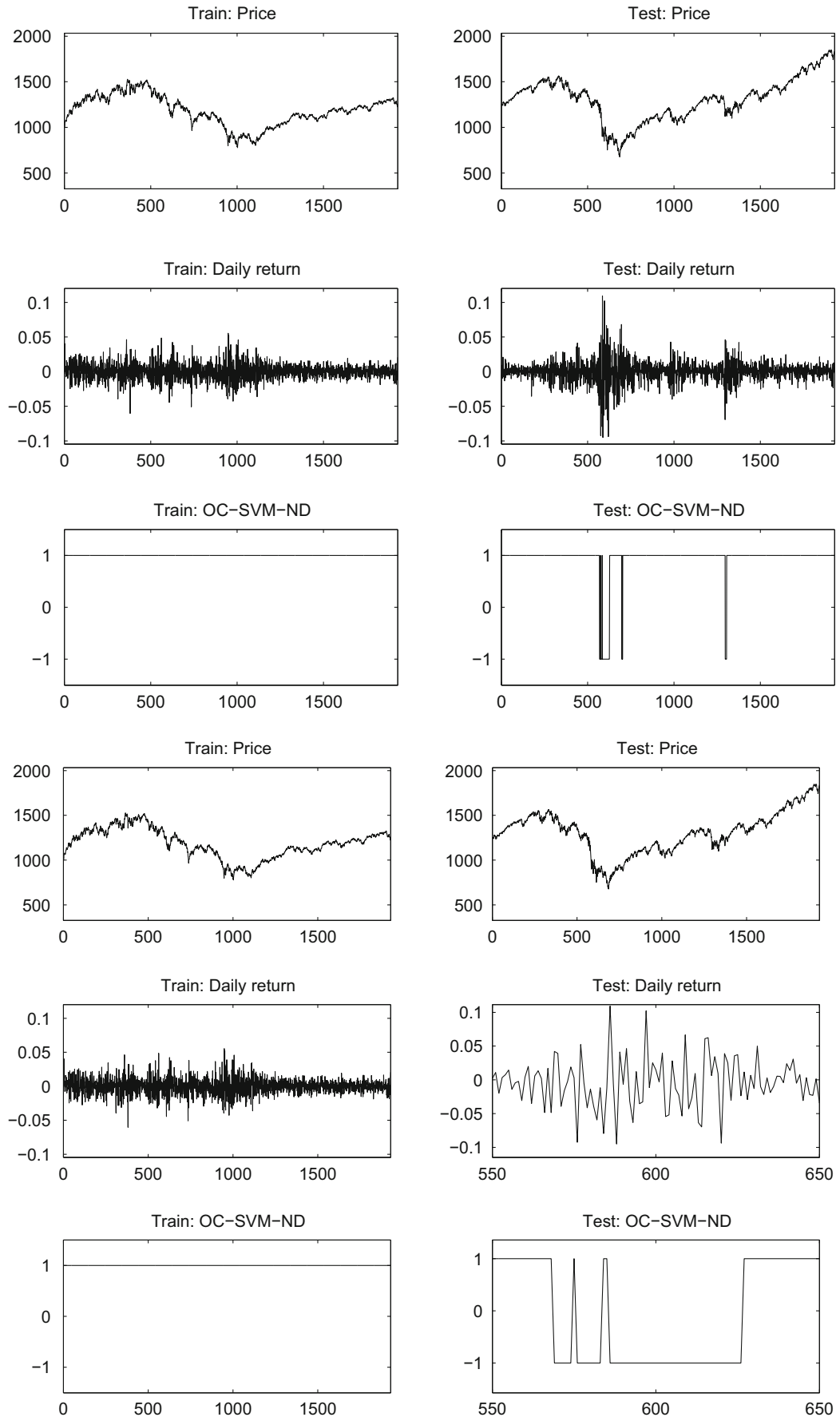

Fig. 9 S\&P500 returns: OC-SVM-ND $(6,8,5)$; bottom figure is identical to the top one with magnified bottom charts (Test: Daily return and Test: $O C-S V M-N D$ ) to demonstrate the early novelty detection by the algorithm 

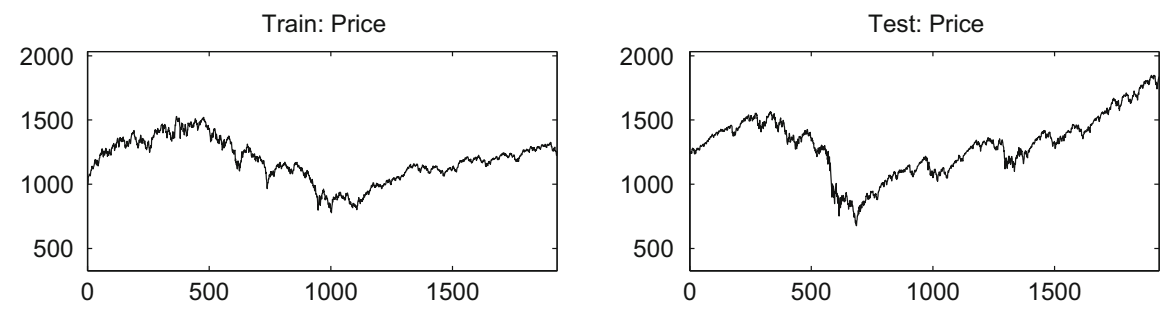

Train: Daily return
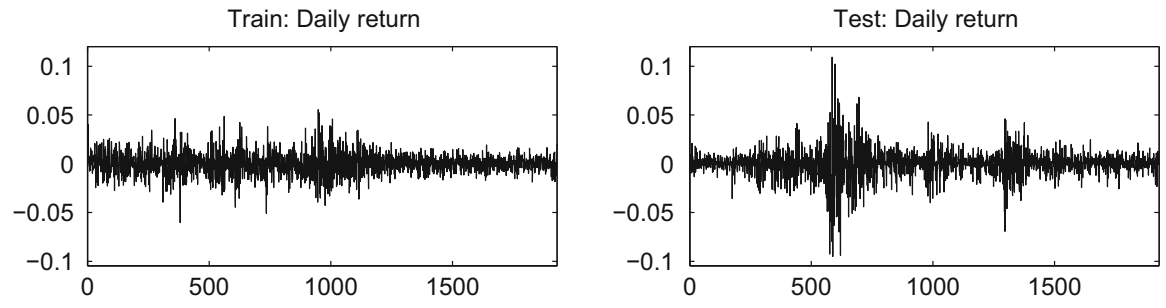

Train: OC-SVM-ED
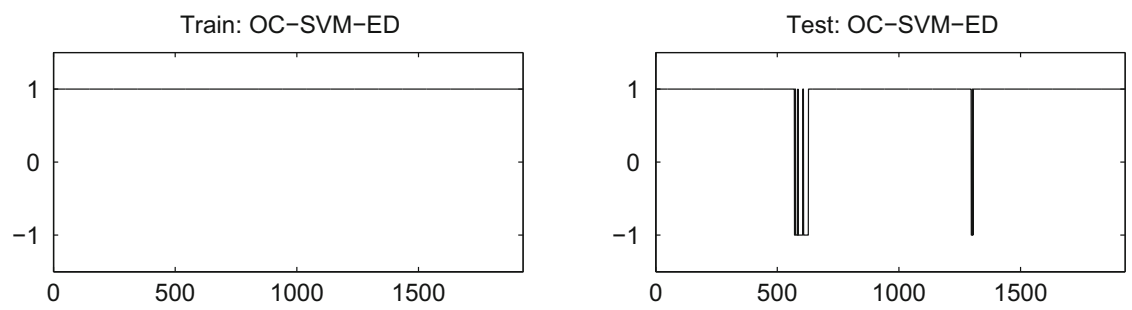

Fig. 10 S\&P500 returns: OC-SVM-ED $(7,7,7)$ 

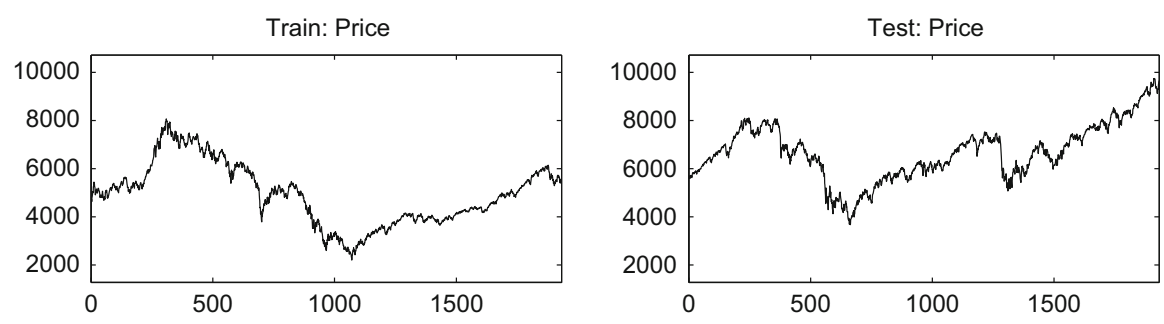

Train: Daily return

Test: Daily return
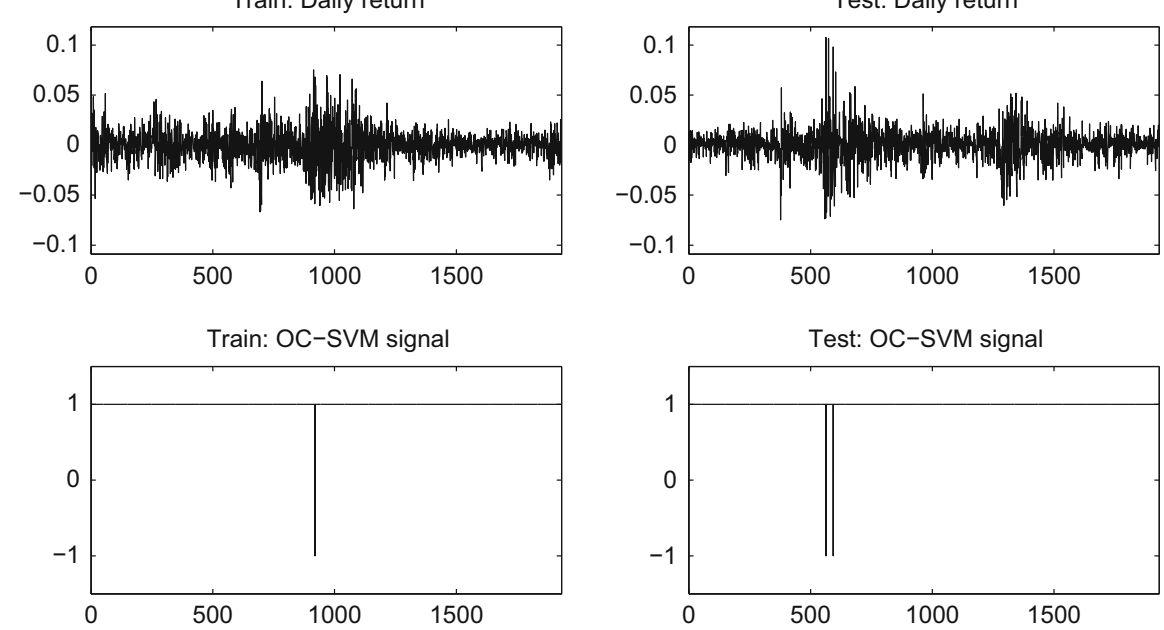

Fig. 11 DAX returns: OC-SVM-ND $(1,6,8)$ 

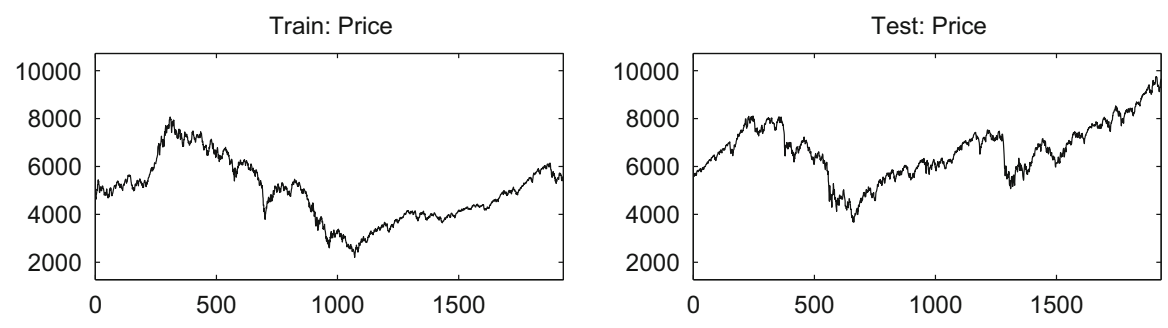

Train: Daily return

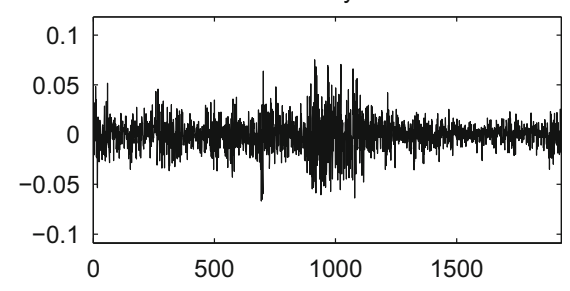

Test: Daily return

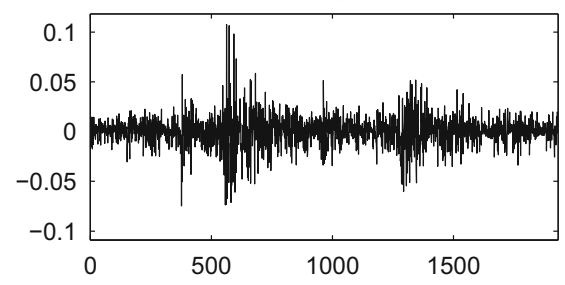

Train: OC-SVM signal
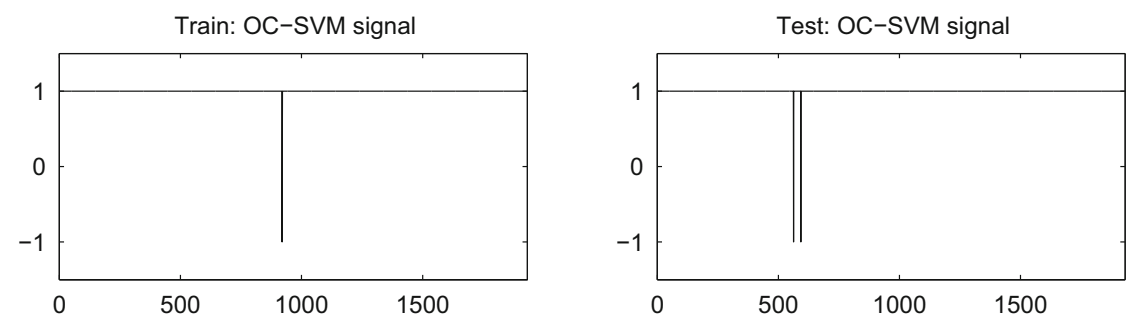

Fig. 12 DAX returns: OC-SVM-ED $(1,6,8)$ 

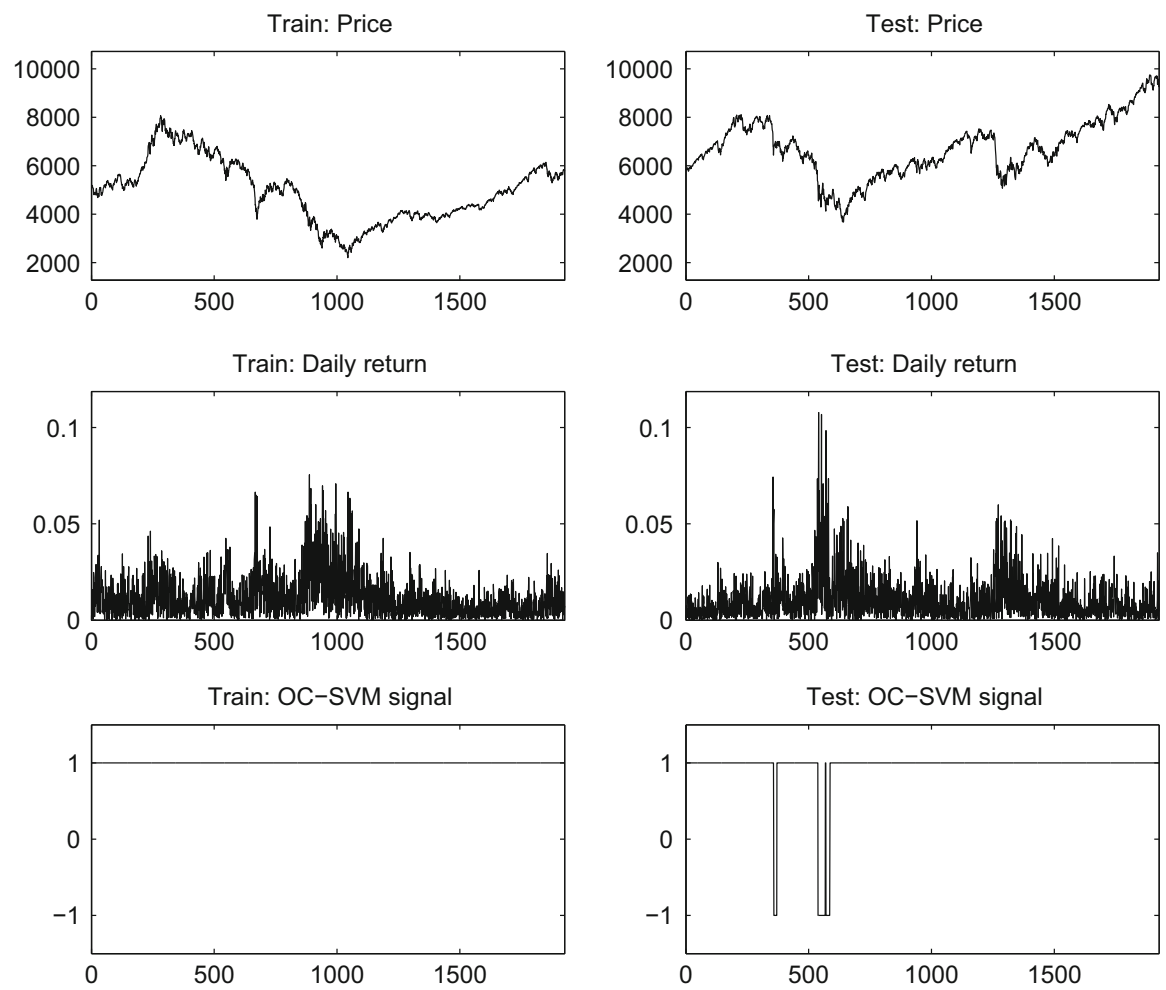

Fig. 13 DAX absolute returns: OC-SVM-ND $(15,9,4)$ 

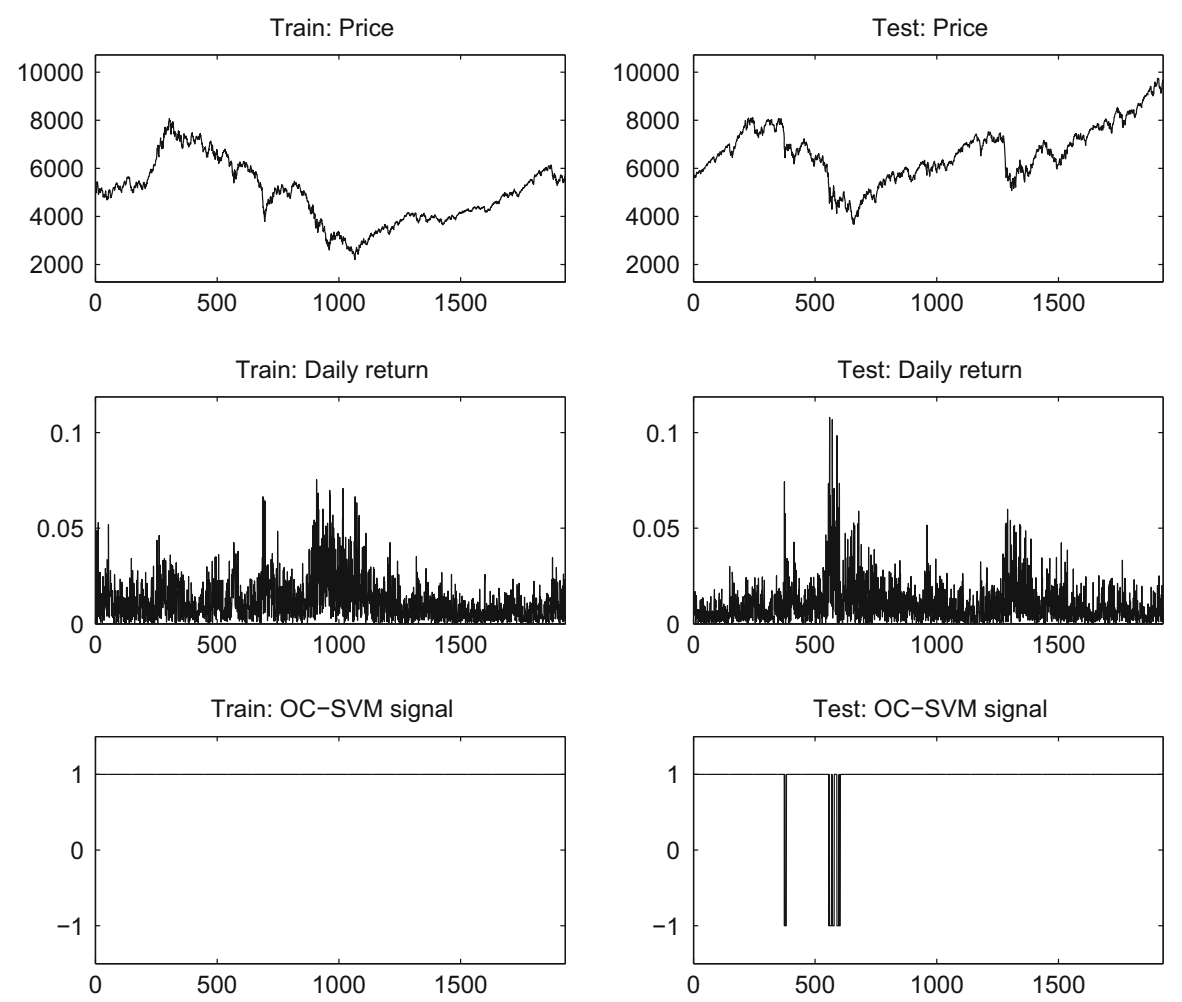

Fig. 14 DAX absolute returns: OC-SVM-ED $(7,8,6)$ 

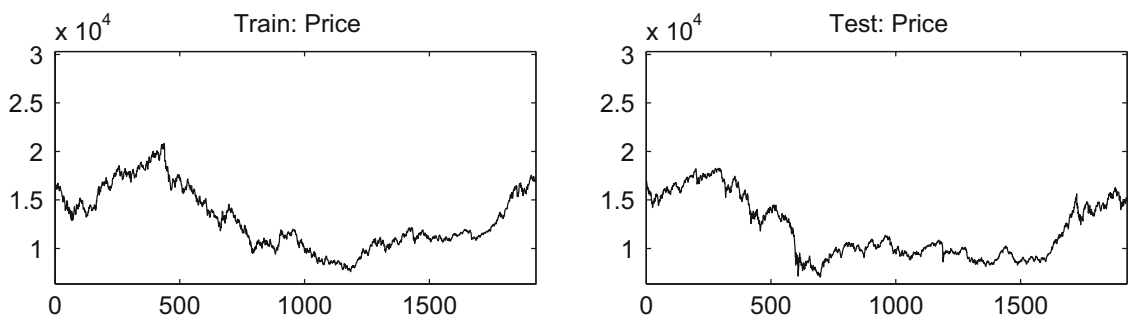

Train: Daily return

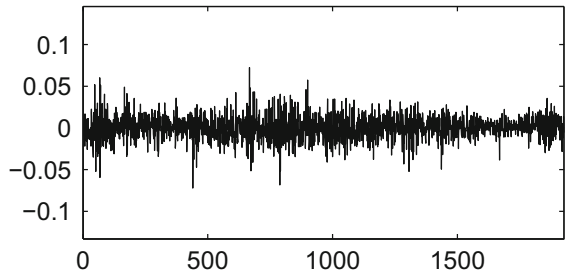

Test: Daily return

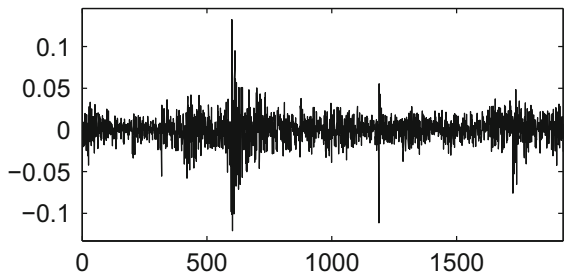

Train: OC-SVM-ND

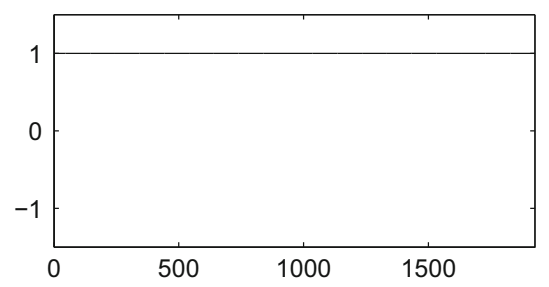

Test: OC-SVM-ND

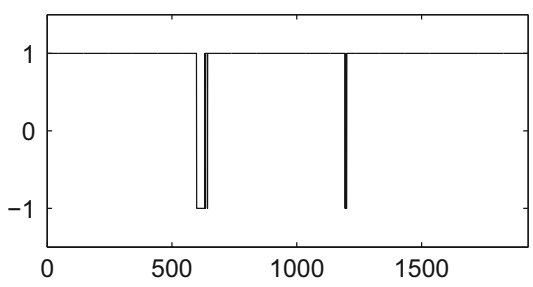

Fig. 15 NIKKEI225 returns: OC-SVM-ND $(16,9,1)$ 

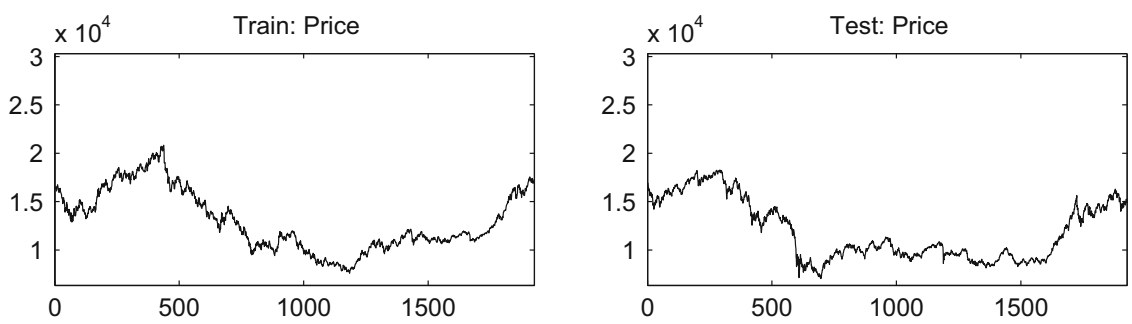

Train: Daily return

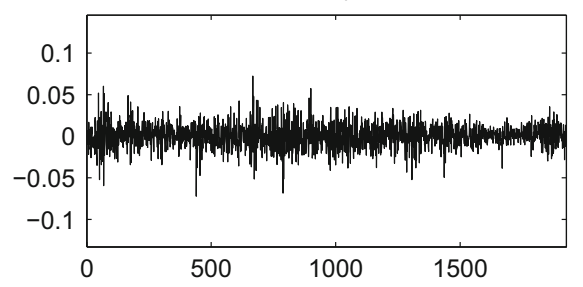

Test: Daily return

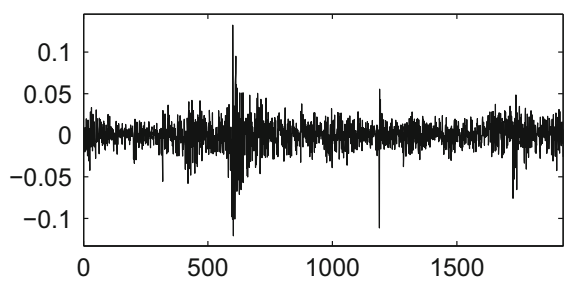

Train: OC-SVM-ED

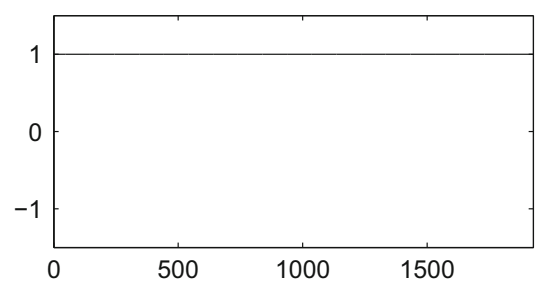

Test: OC-SVM-ED

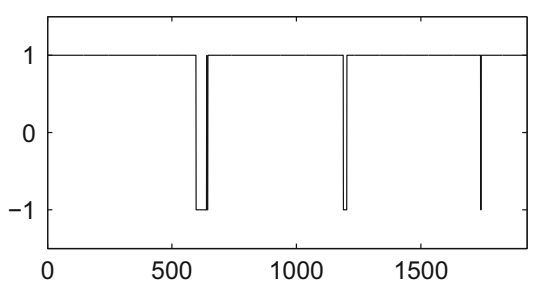

Fig. 16 NIKKEI225 returns: OC-SVM-ED $(16,9,1)$ 

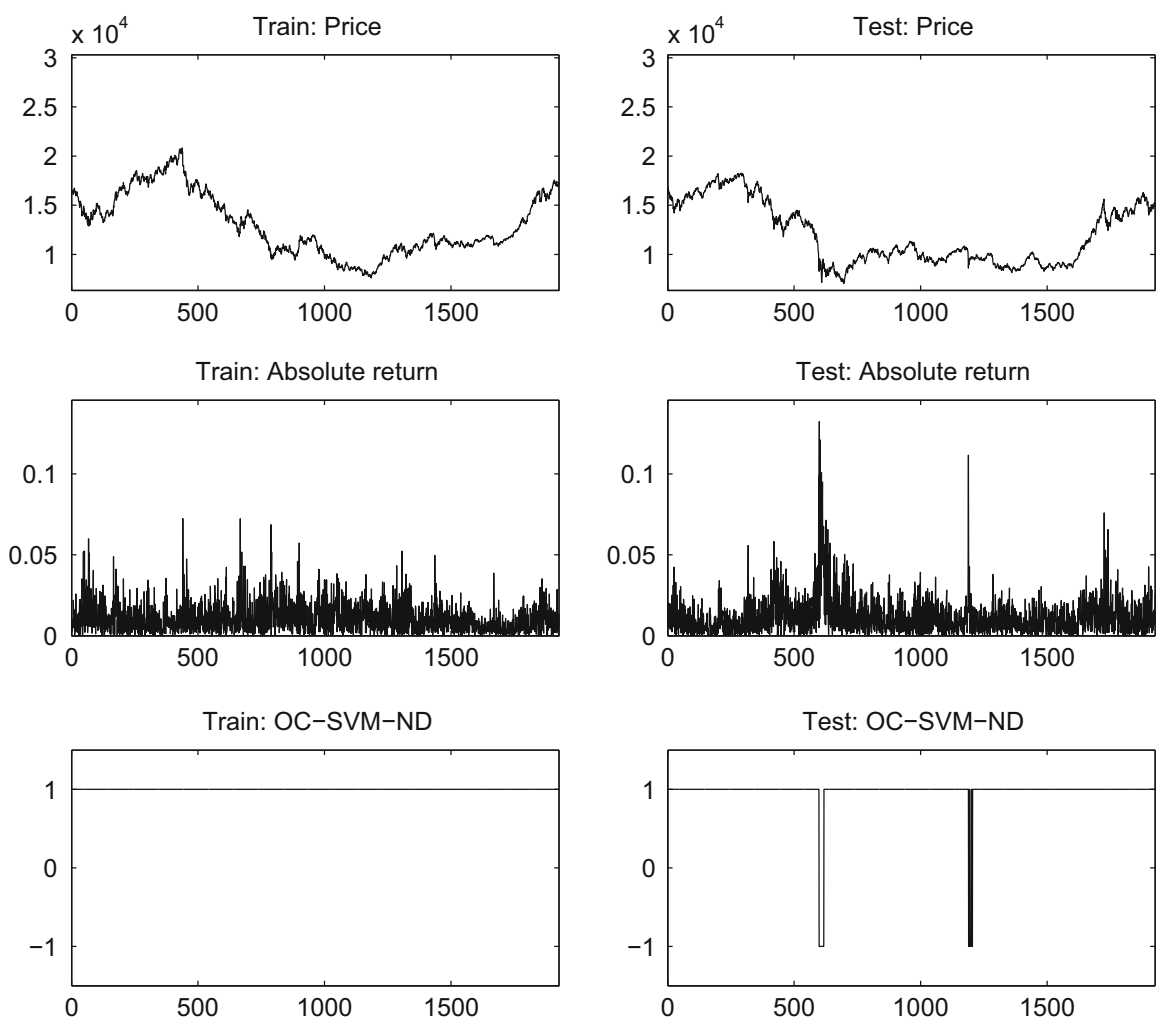

Fig. 17 NIKKEI225 absolute returns: OC-SVM-ND $(16,9,1)$ 

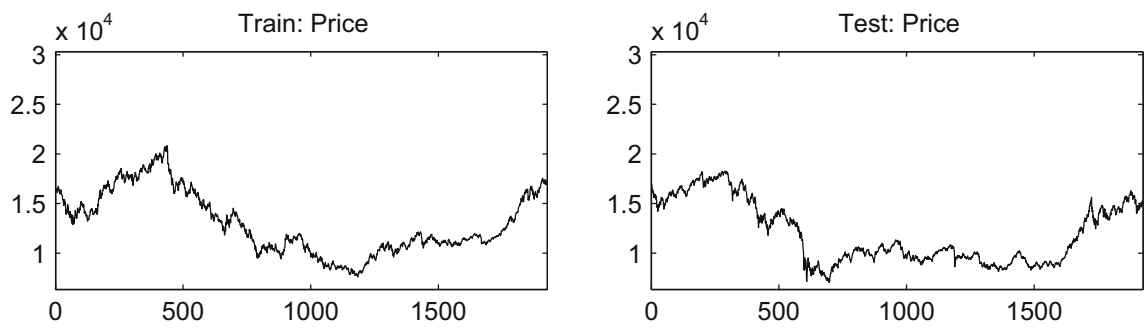

Train: Absolute return

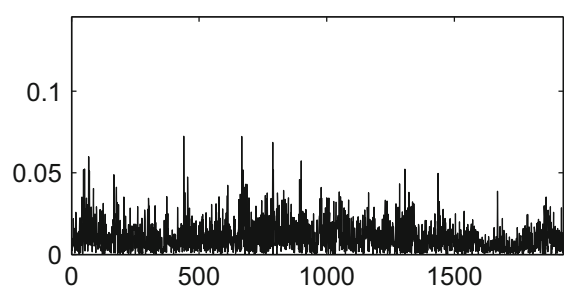

Test: Absolute return

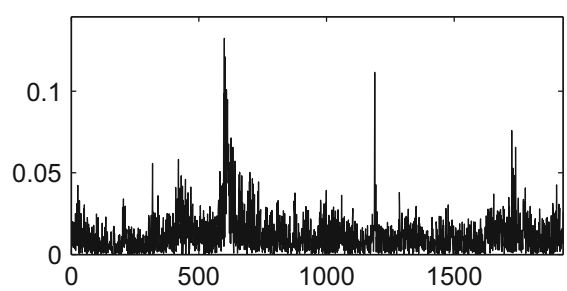

Train: OC-SVM-ED
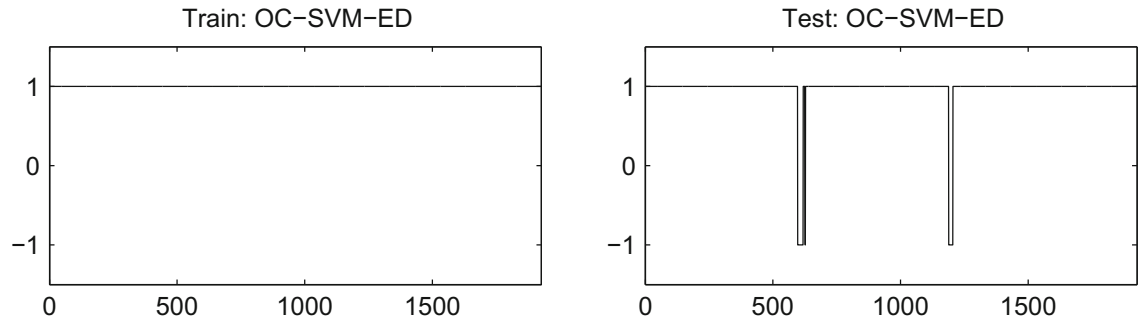

Fig. 18 NIKKEI225 absolute returns: OC-SVM-ED $(16,9,1)$

\section{References}

1. Cristianini, N., Shawe-Taylor, J.: Kernel Methods for Pattern Analysis. Cambridge University Press, Cambridge (2004)

2. Ma, J., Perkins, S.: Time-series novelty detection using one-class support vector machines. In: Proceedings of the International Joint Conference on Neural Networks (2003)

3. Vert, R., Vert, J-P.: Consistency and convergence rates of one-class SVMs and related algorithms. J. Mach. Learn. Res. 7, 815-854 (2006) 